\section{(C) OPEN ACCESS}

\title{
Associations of depression and anxiety symptoms with sexual behaviour in women and heterosexual men attending sexual health clinics: a cross- sectional study
}

\author{
Rachel Margaret Coyle, ${ }^{\oplus 1}$ Fiona C Lampe, ${ }^{1}$ Ada Rose Miltz, ${ }^{\oplus 1}$ Janey Sewell, ${ }^{\oplus 1}$ \\ Jane Anderson, ${ }^{2}$ Vanessa Apea ${ }_{1}^{3}$ Simon Collins, ${ }^{4}$ Rageshri Dhairyawan, ${ }^{5}$ \\ Anne M Johnson, ${ }^{1}$ Monica Lascar, ${ }^{3}$ Sue Mann, ${ }^{6}$ Rebecca O'Connell, ${ }^{3}$ Lorraine Sherr, ${ }_{1}^{1}$ \\ Andrew Speakman, ${ }^{1}$ Alan Tang, ${ }^{7}$ Andrew N Phillips, ${ }^{1}$ Alison Rodger, ${ }^{1}$ on behalf of the \\ AURAH Study Group
}

${ }^{1}$ Institute for Global Health, University College London, Royal Free Hospital, London, UK ${ }^{2}$ Centre for the Study of Sexual Health and HIV, Homerton University Hospital NHS Foundation Trust, London, UK ${ }^{3}$ Ambrose King Centre, Barts Health NHS Trust, London, UK ${ }^{4}$ HIV i-Base, London, UK ${ }^{5}$ Outpatients East, Barking, Havering and Redbridge University Hospitals NHS Trust, London, UK

${ }^{6}$ Camberwell Sexual Health Centre, King's College Hospital, London, UK

${ }^{7}$ Royal Berkshire Hospital NHS Foundation Trust, Reading, UK

\section{Correspondence to} Dr Rachel Margaret Coyle, Institute for Global Health, University College London, London NW3 2PF, UK: rachelcoyle@nhs.net

Received 16 May 2018 Revised 30 November 2018 Accepted 27 December 2018 Published Online First 27 February 2019

Check for updates

\section{(c) Author(s) (or their} employer(s)) 2019. Re-use permitted under CC BY-NC. No commercial re-use. See rights and permissions. Published by BMJ.

\footnotetext{
To cite: Coyle RM, Lampe FC, Miltz AR, et al. Sex Transm Infect 2019;95:254-261.
}

\section{ABSTRACT}

Objective To assess the association of symptoms of depression and anxiety with sexual risk behaviour and history, among women and heterosexual men attending genitourinary medicine (GUM) clinics.

Methods Attitudes to and Understanding of Risk of Acquisition of HIV (AURAH) was a cross-sectional, self-administered questionnaire study recruited from 20 GUM clinics in England, 2013-2014. This analysis included women and heterosexual men. The prevalence of depression and anxiety symptoms was assessed. Modified Poisson regression was used to produce adjusted prevalence ratios (aPR) for the association of $t$ demographic, socioeconomic and lifestyle factors with depression and anxiety, adjusted for gender, age, ethnicity, education level and study region. Among individuals reporting sex in the past 3 months, associations of depression and anxiety with sexual risk behaviour and history were assessed separately by gender, adjusted for age, ethnicity, study region, education and relationship status.

Results Questionnaires were completed by 676 women and 470 heterosexual men. Depression symptoms were reported by 100 (14.8\%) women and 33 men (7.0\%). Anxiety symptoms were reported by 79 women $(11.7 \%)$ and 21 men (4.5\%). Among women reporting recent sex, those with depression symptoms were more likely to report condomless sex with a non-regular partner, aPR 1.38 (1.07-1.77) and recent condomless sex with two or more partners, 1.80 (1.25-2.59). Women with anxiety symptoms more likely to report recent condomless sex with two or more partners, 1.68 (1.13-2.50), low self-efficacy for condom use, 1.54 (1.02-2.31) and STI diagnosis in the last year 1.51 (1.04-2.20). Among heterosexual men reporting recent sex, depression and anxiety symptoms were associated with low self-efficacy with condom use, $2.32(1.29-4.19)$ for depression and 2.23 (1.26-3.94) for anxiety, but not with measures of condomless sex.

Discussion The associations between psychological symptoms and sexual risk behaviours highlight the importance of holistic assessment of need by both general and sexual health clinicians. We highlight the challenge in delivering holistic care associated with fragmentation of sexual health services.

\section{INTRODUCTION}

There is a strong association between depression and risk factors for chronic illness, for example, smoking, alcohol ${ }^{1-3}$ and between depression and chronic disease. ${ }^{4}$ However, establishing a causal relationship between mental and physical illness is complicated by difficulties in establishing temporality. ${ }^{1}$ A similar link between mood disorder and sexual health has been suggested. ${ }^{5}$ In a nationally representative UK survey, men and women reporting treatment for depression were significantly more likely to report condomless sex with two or more partners and to perceive themselves to be at high risk of STI compared with individuals with no symptoms of, and not on treatment for, depression. ${ }^{5}$ There is also evidence from the $\mathrm{UK}^{6}$ and the $\mathrm{USA}^{7}$ that symptoms of poor mental health may be more common in attenders at sexual health clinics than in the general population.

The purpose of the 'Attitudes to and Understanding of Risk of Acquisition of HIV' (AURAH) study was to understand the characteristics of individuals at risk of STI and HIV infection to inform and target health promotion. This study was carried out in genitourinary medicine (GUM) clinics in England. Individuals attending GUM clinics in England may do so without referral and can be seen for various sexual and reproductive health issues. A number of international studies have analysed the relationship between depression and sexual risk behaviour, however few UK studies have focused on women and heterosexual men attending GUM clinics, and to our knowledge none have assessed the relationship between anxiety and sexual risk. A previous analysis of the AURAH study assessed the association of depressive symptoms with sexual behaviour among men who have sex with men (MSM). ${ }^{8}$ In this paper, we estimate the 
prevalence of depression and anxiety symptoms among women and heterosexual men; assess the association of demographic, socioeconomic and lifestyle factors, with depression and anxiety symptoms, and assess the association of depression and anxiety symptoms with self-reported sexual behaviour and history measures.

\section{METHODS}

The AURAH methods have been described in detail elsewhere. ${ }^{9}$ Briefly, AURAH was a cross-sectional questionnaire study that recruited individuals from 20 GUM clinics across England between June 2013 and November 2014. AURAH inclusion criteria were: HIV negative (or undiagnosed at recruitment), aged 18 years or over, and attending for a routine STI screen and/or HIV testing. Initially, study recruitment was unrestricted, after 6 months recruitment was targeted towards MSM and individuals of black ethnicity to ensure adequate representation of the demographic groups at particular risk of HIV. This analysis uses data collected from women and heterosexual men. Data collected from MSM have been analysed and reported separately. ${ }^{8}$

Participants completed a self-administered questionnaire from which the following information was derived.

\section{Sexual behaviour/history measures}

Variables included: recent (in the previous 3 months) condomless vaginal or anal sex with (1) a non-regular partner/s, (2) $\geq 2$ partners or (3) a partner of unknown or positive HIV status; $\geq 2$ new sexual partners within the previous year; STI diagnosis within the previous year; history of ever testing for HIV and self-efficacy with condom use. Participants were deemed to demonstrate low self-efficacy if they disagreed/strongly disagreed with the statement, 'I feel confident that, if I want to, I can make sure a condom is used during sex with any partner, in any situation,' or if they agreed/strongly agreed with the statement, 'I find it difficult to discuss condom use with any new sexual partner.' For condomless sex measures and STI history, missing data were taken to indicate absence of the behaviour. This assumption was tested in a sensitivity analysis detailed below.

\section{Symptoms of depression and anxiety}

Symptoms of depression and anxiety were assessed using the Patient Health Questionnaire (PHQ9) ${ }^{10}$ and General Anxiety Disorder (GAD7) ${ }^{11}$ screening tools. Participants are asked if they have experienced each PHQ9/GAD7 symptom in the previous 2 weeks (with four possible responses from: 'not at all' to 'more than half the days') coded 0-3. A PHQ9 score of $\geq 10$ (maximum score 27) was used to define depression symptoms, this has been widely validated ${ }^{10}$ and corresponds to moderate-severe symptoms. PHQ2 scores were also calculated based on the first two PHQ9 symptoms to facilitate comparison with similar studies; a score of $\geq 3$ indicates depression symptoms. ${ }^{58}$ A GAD7 score of $\geq 10$ (maximum score 21 ) was used to define anxiety symptoms. ${ }^{11}$ To account for the potential impact of treatment on active symptoms, participants were also asked if they were currently receiving any (medical or other) treatment for depression. Additional mesures of depression were defined in which participants reporting treatment for depression were included as well as those positive for PHQ9/PHQ2.

\section{Demographic, socioeconomic and lifestyle data}

Data included age, gender, ethnicity (White, Black African, Black Caribbean, other), relationship status (ongoing relationship with a partner/spouse - yes/no), study region (London or other), UK birth, employment (employed, student or unemployed/other), housing (home owner, rented or 'unstable accommodation' including temporary accommodation and homeless), financial hardship (participants were asked if they had sufficient money to meet basic needs, possible responses: yes, mostly, sometimes or no), education (university degree or not), recent (in the previous 3 months) recreational drug use and smoking status (current, ex-smoker or non-smoker). Alcohol intake was assessed using the first two questions of the Alcohol Use Disorders Identification Test-Consumption $^{12}$ tool (AUDIT-C): a score of $\geq 6$ is taken to indicate higher risk drinking.

\section{Analysis populations}

The prevalence of depression and anxiety symptoms and their association with demographic, socioeconomic and lifestyle factors is reported in all women and heterosexual men. Individuals reporting no vaginal or anal sex in the last 3 months were excluded from the analyses assessing the associations of symptoms with sexual behaviour/history because previous studies have demonstrated an association between depression and lack of sexual activity, ${ }^{58}$ and we aimed to compare condomless sex with condom-protected sex. Women who identified as gay and those with missing data on sexuality were also excluded from the analysis of sexual behaviour/history; bisexual women were retained in this analysis. Although bisexual women may have unique risks for depression, the small numbers of women reporting that they were bisexual prevented their analysis as a separate group.

\section{Statistical analysis}

The prevalence of depression and anxiety symptoms is presented with $95 \%$ CIs. $\chi^{2}$ tests were used to assess the unadjusted association of demographic, socioeconomic and lifestyle characteristics with these symptoms. Modified Poisson regression models (Poisson models with log link and robust error variance) were used to assess the adjusted associations of each of these characteristics with depression and anxiety symptoms. Each factor was considered separately with adjustment for age group, gender, ethnicity, study region and education. Modified Poisson regression was used to assess the relationship of depression and anxiety symptoms with sexual behaviour/history in individuals reporting recent sex, in women and in heterosexual men separately, with adjustment for age group, ethnicity, study region, education and relationship status. Associations are presented as adjusted prevalence ratios with 95\% CIs (aPR/95\% CI). Modified Poisson regression is an accepted alternative to logistic regression in the analysis of binary outcomes, the main advantage of which is that it estimates the readily interpretable prevalence ratio, rather than the OR. ${ }^{13}$

Statistical analysis was undertaken in Stata V.14.1.

\section{Sensitivity analysis}

The association between psychological symptoms and sexual behaviour/history was explored with additional adjustment for higher risk alcohol use and recent recreational drug use, because previous studies have demonstrated a link between alcohol and drug use and sexual risk behaviour, and we aimed to assess evidence that these factors mediated the association between psychological symptoms and sexual risk behaviour. ${ }^{8}$

An additional sensitivity analysis was carried out excluding participants with missing data on condomless sex and STI history. 


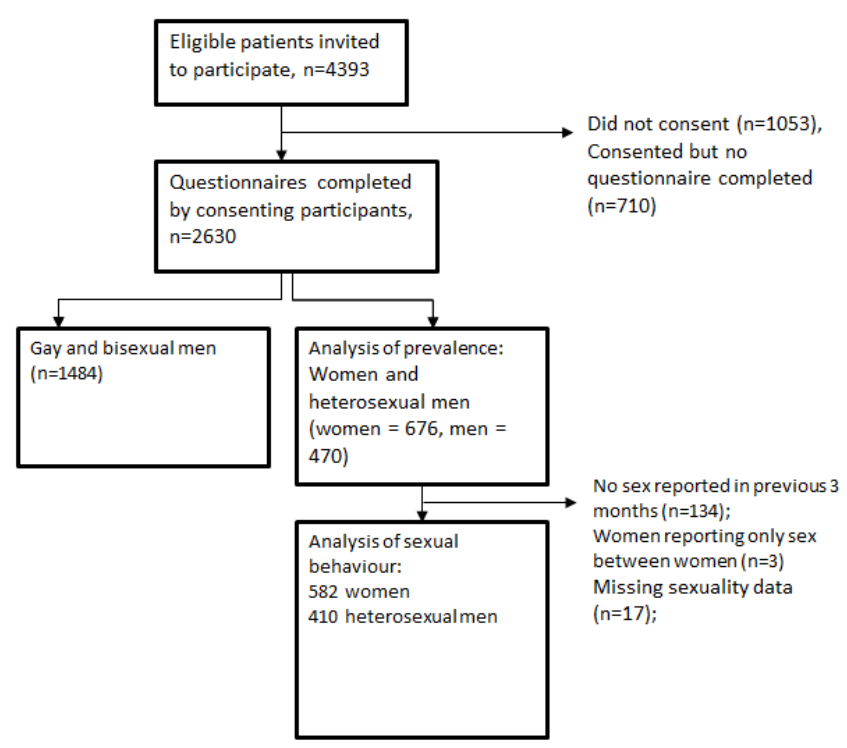

Figure 1 Study recruitment, inclusions and exclusions.

\section{RESULTS}

There were 2630 participants in the AURAH study (response rate 60\%). Following exclusion of 1484 MSM there were 1146 individuals: 470 (41.0\%) heterosexual men and 676 (59.0\%) women. Of the women, 624 of $676(92.3 \%)$ were heterosexual, $32(4.7 \%)$ were bisexual, $3(<1.0 \%)$ were gay and 17 missing $(2.5 \%)$. Figure 1 demonstrates recruitment, inclusions and exclusions.

Table 1 demonstrates the characteristics of all 676 women and 470 heterosexual men in AURAH. The median age of women was 26 years, and of men was 29 years.

\section{Prevalence of depression and anxiety symptoms}

The prevalence of depression symptoms (PHQ9 $\geq 10$ ) was $14.8 \%$ among women and $7.0 \%$ among heterosexual men; corresponding prevalence for anxiety symptoms (GAD7 $\geq 10$ ) was $11.7 \%$ and $4.5 \%$. Symptoms of both depression and anxiety were reported by 62 women (9.2\%) and by 14 men (3.0\%) (table 2).

\section{Demographic, socioeconomic and lifestyle factors: association with depression and anxiety symptoms}

In the unadjusted analysis female gender, non-London study region, not being in a relationship, non-employment, financial hardship and current smoking were associated with depression symptoms (table 3), and there was some evidence of association with non-university education, rented or unstable housing and higher risk drinking. In adjusted models women were more than twice as likely as men to have depression symptoms, individuals not in a relationship were about $70 \%$ more likely to have depression symptom compared with those in a relationship, those reporting the most severe level of financial hardship were almost four times as likely to report depression symptoms as those with sufficient money, depression prevalence was almost twofold higher for current smokers compared with non-smokers, and for those reporting higher risk drikning, and was about 50\% higher for those reporting recent recreational drug use. Associations of study region, education, work situation and housing with depression symptoms were attenuated after adjustment. Age, ethnicity and UK birth were not associated with depression symptoms in unadjusted or adjusted analysis.
Table 1 Demographic and socioeconomic characteristics of women and heterosexual men in AURAH

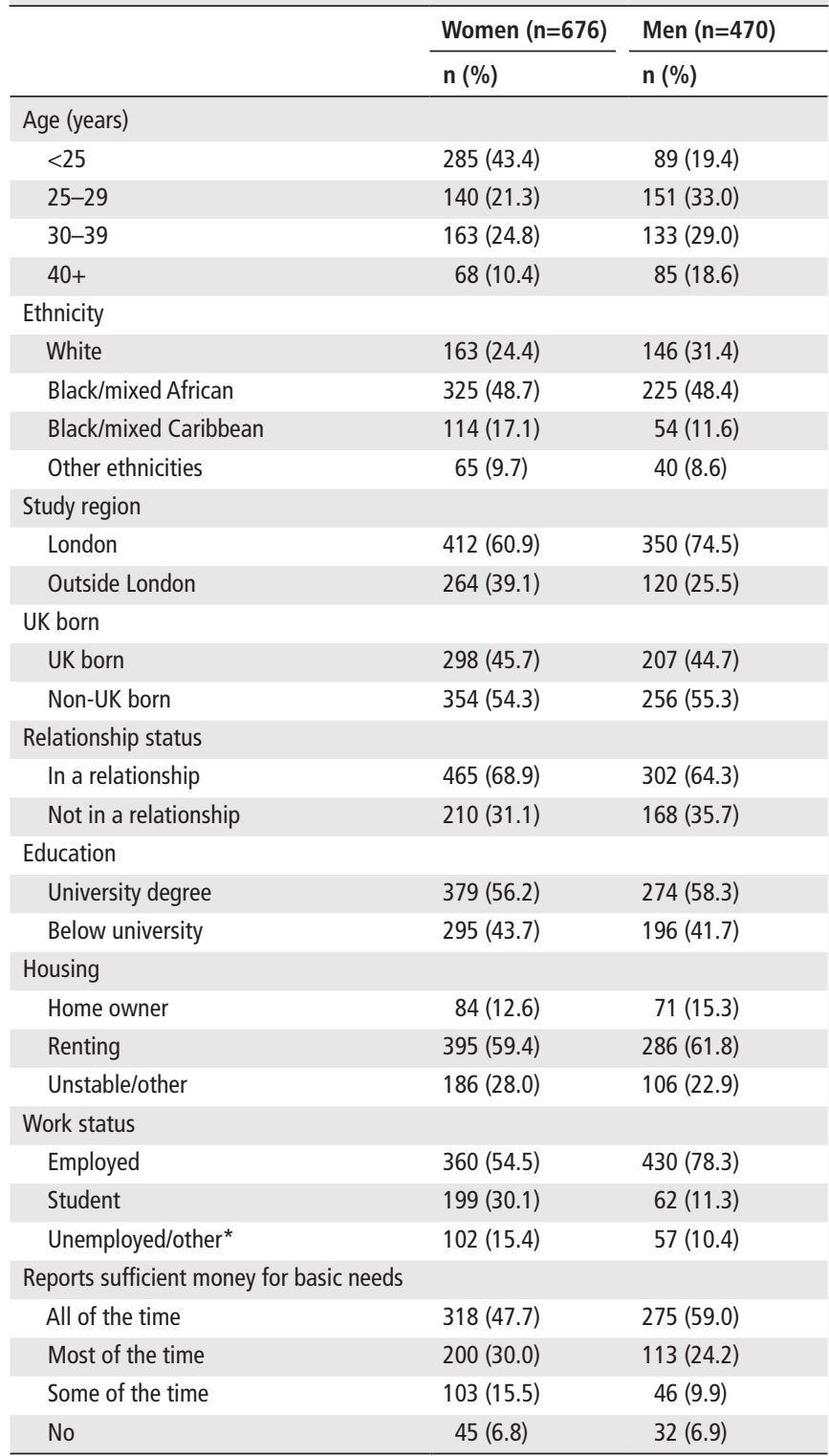

*includes retired and carer.

AURAH, Attitudes to and Understanding of Risk of Acquisition of HIV.

Anxiety symptoms were associated with female gender, unemployment, financial hardship, non-university education and current smoking in unadjusted analyses (table 3). These associations remained in adjusted analysis, except for the association with unemployment which was attenuated. In contrast to depression symptoms, no significant associations with anxiety symptoms were seen for relationship status, recent recreational drug use and higher risk drinking with anxiety symptoms. In addition, age group, ethnicity, study region, UK birth and housing status were not associated with anxiety symptoms.

\section{Treatment for depression}

Current medical or other treatment for depression was reported by $10 / 470$ men, $2.1 \%$ (95\% CI 1.0 to 3.9 ) and $37 / 676$ women, $5.5 \%$ (95\% CI 3.9 to 7.5$)$. In total, 41 men $(8.7 \%, 95 \%$ CI 6.3 to 11.6$)$ and $122(18.0 \%, 95 \%$ CI 15.2 to 21.2$)$ women reported depression symptoms (PHQ9 $\geq 10$ ) and/or current treatment, and 39 men, $8.3 \%$ (95\% CI 6.0 to 11.2$)$ and 126 women, $18.6 \%$ 
Table 2 Prevalence of depression and anxiety symptoms in women and heterosexual men

\begin{tabular}{|c|c|c|c|c|c|}
\hline & & \multicolumn{2}{|c|}{ Women $n=676$} & \multicolumn{2}{|c|}{ Heterosexual men $n=470$} \\
\hline & & $\mathbf{n}$ & $\%(95 \% \mathrm{Cl})$ & $\mathrm{n}$ & $\%(95 \% \mathrm{Cl})$ \\
\hline \multicolumn{6}{|l|}{ PHQ9 score } \\
\hline $0-4$ & No/minimal & 451 & 66.7 (63.0 to 70.2$)$ & 362 & 77.0 (72.9 to 80.8$)$ \\
\hline $5-9$ & Mild & 125 & 18.5 (15.7 to 21.7$)$ & 75 & 16.0 (12.8 to 19.6$)$ \\
\hline $10-14$ & Moderate & 45 & $6.7(4.9$ to 8.8$)$ & 24 & $5.1(3.3$ to 7.5$)$ \\
\hline $15-19$ & Moderate-severe & 41 & $6.1(4.4$ to 8,2$)$ & 5 & $1.1(0.3$ to 2.5$)$ \\
\hline $20-27$ & Severe & 14 & 2.1 (1.1 to 3.5$)$ & 4 & $0.9(0.2$ to 2.2$)$ \\
\hline PHQ9 score $\geq 10$ & Depression symptoms & 100 & $14.8(12.2$ to 17.7$)$ & 33 & 7.0 (4.9 to 9.7$)$ \\
\hline PHQ2* score $\geq 3$ & Depression symptoms & 100 & $14.8(12.2$ to 17.7$)$ & 31 & 6.6 (4.6 to 9.3$)$ \\
\hline GAD7 score $\geq 10$ & Anxiety symptoms & 79 & 11.7 (9.4 to 14.4$)$ & 21 & 4.5 (2.9 to 6.9$)$ \\
\hline PHQ9 $\geq 10$ and GAD7 $\geq 10$ & Both depression and anxiety symptoms & 62 & $9.2(7.2$ to 11.7$)$ & 14 & $3.0(1.7$ to 5.1$)$ \\
\hline
\end{tabular}

*Based on first two PHQ9 questions.

GAD, General Anxiety Disorder; PHQ, Patient Health Questionnaire.

(95\% CI 15.8 to 21.8 ) had depression symptoms by PHQ2 (PHQ2 $\geq 3$ ) and/or current treatment.

\section{Association of depression and anxiety symptoms with sexual behaviours and sexual history}

Sex in the last 3 months was reported by 582/676 (86.1\%, 83.2-88.6) women and $410 / 470(87.2 \%, 83.7-90.0)$ men. The prevalence of each sexual behaviour/history measure and the adjusted association of depression and anxiety symptoms with these measures are shown in table 4, among 582 women and 410 men who reported sex in the last 3 months (see figure 1).

After adjustment, there was some evidence of an association between depression symptoms and condomless sex in women, but little evidence of a similar association in men.

Women with depression symptoms were more likely to report recent condomless sex with a non-regular partner, aPR/95\% CI 1.38 (1.07 to 1.77), and recent condomless sex with two or more partners 1.80 (1.25 to 2.59). In women, there was no evidence of an association between depression symptoms and other sexual behaviour/history measures including low self-efficacy negotiating condom use, reporting two or more new partners in the last year, or STI diagnosis in the previous year (see table 4).

Among men, after adjustment there was no significant relationship between depression symptoms and the sexual behaviour/history measures with the exception of low self-efficacy negotiating condom use, 2.32 (1.29-4.19).

Among women, anxiety symptoms were associated with reporting recent condomless sex with two or more partners, 1.69 (1.13-2.50), low self-efficacy negotiating condom use, 1.54 (1.02-2.31) and with history of STI diagnosis in the previous year, $1.51(1.04-2.20)$.

In men, anxiety symptoms were associated with low self-efficacy in relation to condom use, 2.23 (1.26-3.94) but not with any other sexual behaviour or history measures.

\section{Sensitivity analysis}

All reported associations between depression symptoms and anxiety symptoms, and sexual risk behaviours remained after additional adjustment for drug and alcohol use. Among women, effect sizes of depression and anxiety symptoms were somewhat attenuated for condomless sex with a non-regular partner and condomless sex with multiple partners (see online supplementary information). In men, the effect size of depression symptoms was slightly increased for low self-efficacy after adjustment for alcohol and drug use. These results suggest that alcohol and drug use influence, and may mediate, sexual risk behaviour but are not likely to be the sole mediators.

Exclusion of participants with missing data for condomless sex and STI history variables did not materially change the results.

\section{DISCUSSION \\ Principal findings}

In this study of women and heterosexual men attending GUM clinics, symptoms of depression were reported by $14.8 \%$ of women and $7.0 \%$ of men and symptoms of anxiety were reported by $11.7 \%$ of women and $4.5 \%$ of men. There was a strong positive association between financial hardship and psychological symptoms, and a positive association between depression symptoms and measures of condomless sex in women. In addition, we report an association of anxiety symptoms with condomless sex, low self-efficacy for condom use and previous STI diagnosis in women. Among men, depression and anxiety were associated with low self-efficacy for condom use but not with other measures of sexual behaviour/history.

\section{Strengths and weaknesses}

A strength of this study is the use of validated screening tools to assess depression and anxiety symptoms and the collection of detailed sexual behaviour data. Our work contributes to the evidence base on the association between psychological symptoms and sexual risk with a focus on individuals attending GUM clinics.

There are some important limitations. It is not possible in a cross-sectional study to determine causality or the direction of the association between psychological symptoms and sexual behaviour. It might reasonably be expected that anxiety symptoms could be provoked by, for example, recent high-risk sexual behaviour. However, the PHQ9 and GAD7 tools are validated for depression and anxiety screening and are intended to capture ongoing, clinically significant symptoms. ${ }^{10}{ }^{11}$ Irrespective of the direction of association, in our study depression and/or anxiety symptoms were reported frequently and we demonstrate an association between these symptoms and some sexual risk behaviours. This suggests holistic assessment of sexual and mental health is likely to be useful in determining risk of poor sexual health. The measurement of sexual behaviours relies on self-report and may therefore be subject to recall error and bias, however it is likely that GUM clinic attenders would have good recall of recent sexual activity. Social desirability bias may influence reporting, and non-participation may have biased estimates of prevalence 
Table 3 Demographic, socioeconomic and lifestyle factors and association with depression and anxiety symptoms in women and heterosexual men

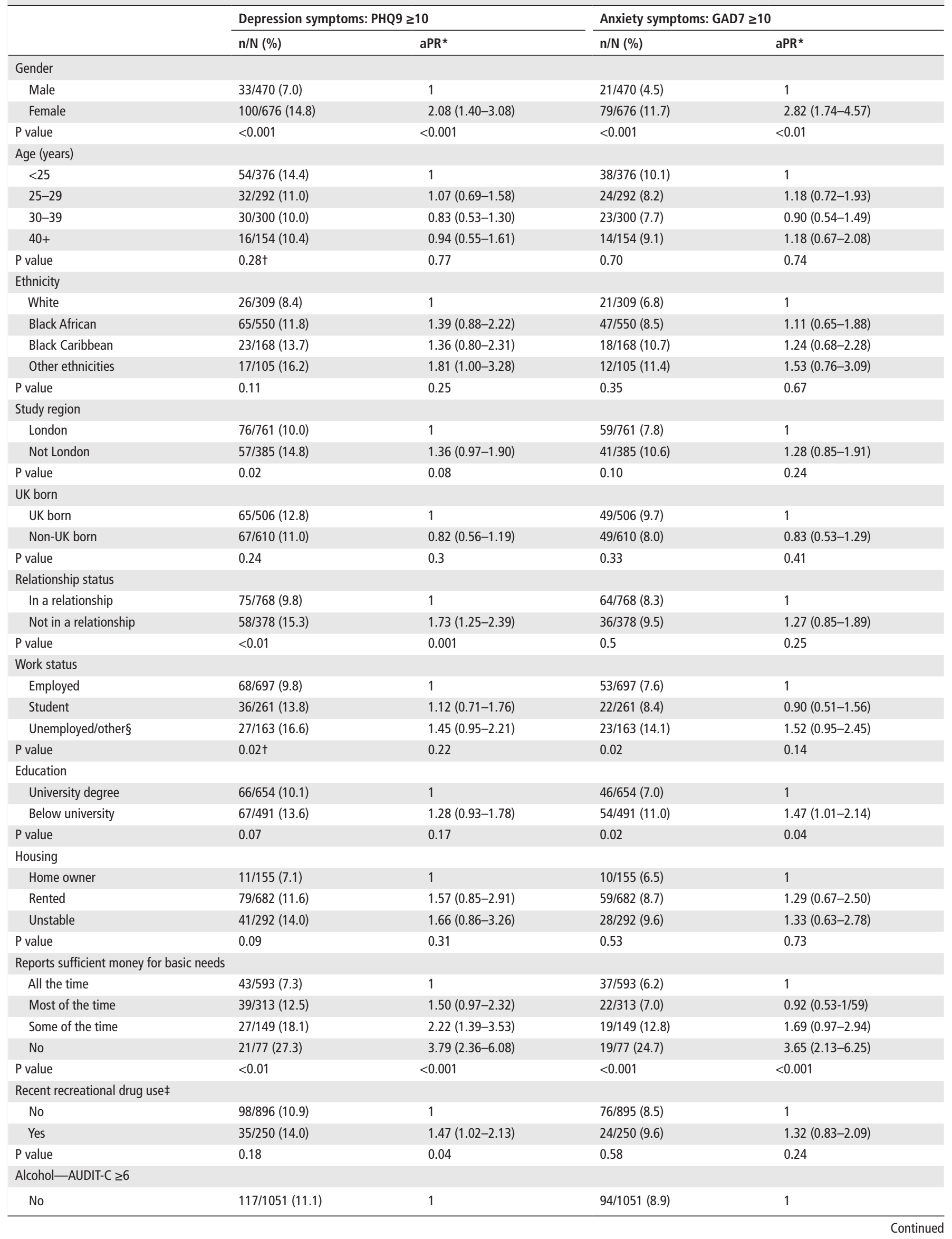


Table 3 Continued

\begin{tabular}{|c|c|c|c|c|}
\hline & \multicolumn{2}{|c|}{ Depression symptoms: PHQ9 $\geq 10$} & \multicolumn{2}{|c|}{ Anxiety symptoms: GAD7 $\geq 10$} \\
\hline & $\mathrm{n} / \mathrm{N}(\%)$ & $\mathrm{aPR}^{*}$ & $\mathrm{n} / \mathrm{N}(\%)$ & $\mathrm{aPR}^{*}$ \\
\hline Yes & $16 / 95(16.8)$ & $1.95(1.20-3.16)$ & $6 / 95(6.3)$ & $0.89(0.34-2.05)$ \\
\hline$P$ value & 0.1 & $<0.01$ & 0.39 & 0.79 \\
\hline \multicolumn{5}{|l|}{ Smoking status } \\
\hline Non-smoker & 68/664 (10.2) & 1 & $55 / 664(8.3)$ & 1 \\
\hline Ex-smoker & 20/212 (9.4) & $1.06(0.66-1.70)$ & $11 / 212(5.2)$ & $0.71(0.38-1.33)$ \\
\hline Current smoker & $45 / 248(18.1)$ & $1.92(1.32-2.79)$ & $32 / 248(12.9)$ & $1.59(1.01-2.46)$ \\
\hline$P$ value & $<0.01$ & $<0.01$ & 0.01 & 0.02 \\
\hline
\end{tabular}

*Adjusted for age, gender, ethnicity, education and study region.

tTest for linear trend.

¥Within the last 3 months.

§Other includes unable to work due to long-term health condition, carers and retired.

AUDIT-C, Alcohol Use Disorders Identification Test-Consumption; GAD, General Anxiety Disorder; PHQ, Patient Health Questionnaire; aPR, adjusted prevalence ratio.

of depression and anxiety. These biases may operate differently in women and heterosexual men, which could contribute to differences in results. Finally, the relatively small number of women and men reporting symptoms of both depression and anxiety prevented analysis of the association between comorbid depression and anxiety on sexual risk behaviour.

\section{Comparison with the literature}

In Natsal-3, Field et al considered reported depression symptoms (PHQ2 score $\geq 3$ ) and pharmacological treatment for depression in estimating the prevalence of depression. They reported an overall prevalence of depression as $18.2 \%$ in women and $11.9 \%$ in men. ${ }^{5}$ This compares to $18.7 \%$ of women in AURAH and $8.3 \%$ of men who had (PHQ2 $\geq 3$ ) for depression and/ or treatment. The definition of treatment within the AURAH study included 'medical therapy' in addition to pharmacological treatment, this would be expected to result in somewhat higher estimations of depression prevalence. Consistent with previous prevalence studies, ${ }^{514} 15$ we demonstrated that depression and anxiety symptoms were more common among women than men, and were strongly associated with some markers of social deprivation. It is not possible to determine causality, however our study supports a relationship between poverty and psychological symptoms which may operate in both directions. Clinicians should consider deprivation when assessing patients, and holistic services should support people in accessing financial support/ benefits as appropriate.

Among individuals who reported recent sex, depression symptoms were associated with condomless sex with a non-regular partner, and condomless sex with two or more partners in women, but not in men. Our findings are broadly consistent with evidence from cross-sectional and longitudinal studies from the USA, ${ }^{16-22}$ Canada $^{23}$, and Eastern Europe ${ }^{22}$ that depression symptoms are associated with condomless sex ${ }^{192024}$ reporting multiple sexual partners ${ }^{17} 1821$ and history of STI diagnosis. ${ }^{18} 2223$ Our study demonstrates that the association between psychological symptoms and sexual risk behaviour is not limited to depression symptoms. We report a positive association between symptoms of anxiety and certain sexual risk behaviours in women. This is important because although we have demonstrated some overlap

Table 4 Association of depression and anxiety symptoms with sexual behaviour and history measures in women and heterosexual men reporting sex in the last 3 months

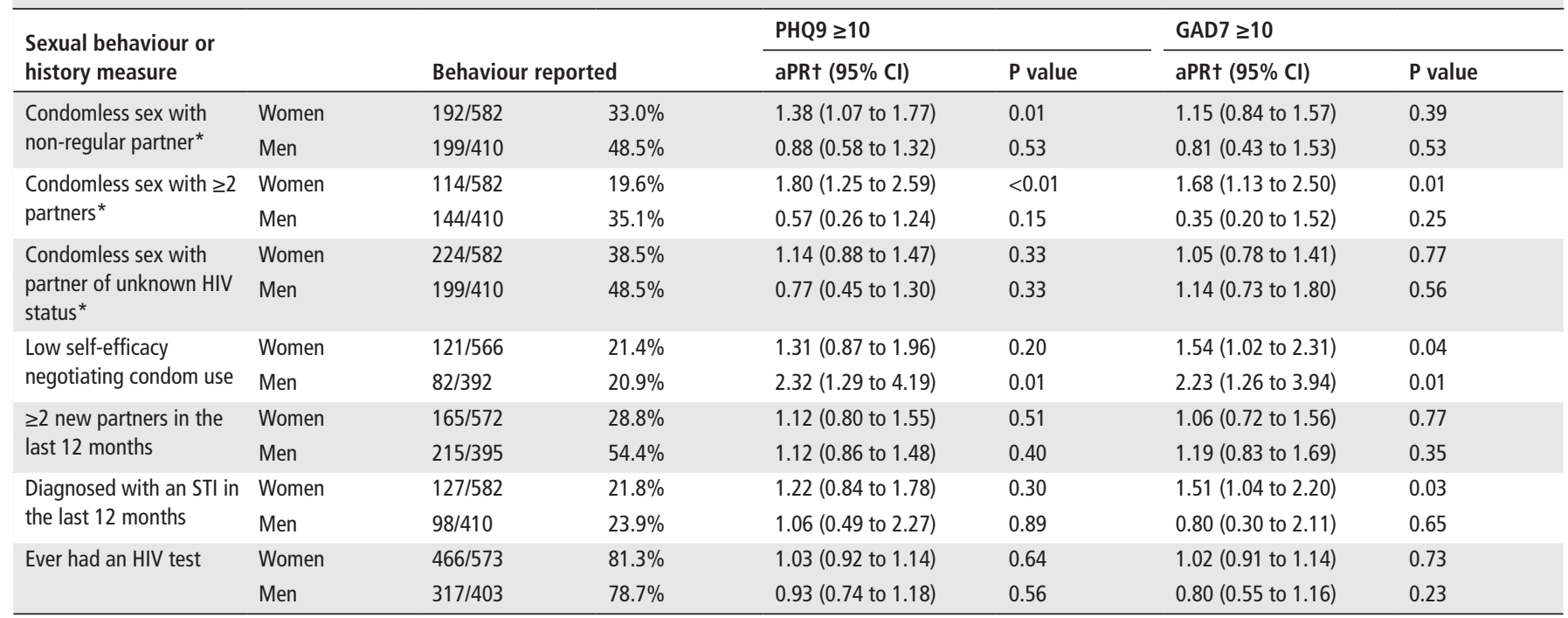

For exclusions see Figure 1.

*Within the last 3 months.

†Adjusted for age, ethnicity, study region, education and relationship status.

GAD, General Anxiety Disorder; PHQ, Patient Health Questionnaire; aPR, adjusted prevalence ratio. 
between symptoms of depression and anxiety, a number of individuals reported symptoms of anxiety only. Women in this group may be at increased risk of STI. Finally, we demonstrated an association between psychological symptoms and self-efficacy regarding condom use in women and heterosexual men. This finding is consistent with our previous analysis of MSM AURAH study participants. ${ }^{8}$

\section{Interpretation and implications}

There are a number of possible explanations for our findings. Common mental health disorders may be associated with impaired judgement and risk evaluation ${ }^{25}$ and have been linked to sexual impulsivity ${ }^{26}$ which may contribute to taking sexual risks. Associations of depression and anxiety with low self-efficacy for condom use may suggest that low self-efficacy is an important mechanism in the link between psychological symptoms and sexual risk. Factors such as intimate partner violence were not measured in this study but may also impact on the dynamics between socioeconomic hardship, psychological symptoms and sexual risk behaviour among women. It is plausible that for all the associations described causality may operate in either direction and this is especially important to consider in the setting of a GUM clinic.

Our findings have implications for clinicians practising in specialist (GUM, mental health) and general (e.g. primary care) settings. Clinicians managing people with mental health problems should consider sexual health needs, and offer testing or signposting as needed. In GUM settings, screening for psychological symptoms may be useful in estimating risk of poor sexual health. ${ }^{5}$ In patients at moderate to high risk of HIV infection, the additional risk conferred by mental illness should be considered when evaluating the role for preventive measures such as pre-exposure prophylaxis against HIV infection, alongside an assessment of barriers to adherence and adherence support strategies. GUM clinicians are skilled in supporting STI risk reduction strategies. Assessment of psychological symptoms in GUM settings may be particularly useful when working with service users presenting with multiple and/or repeated behaviours associated with risk of STI and HIV acquisition.

In England, there are particular challenges in the delivery of holistic sexual healthcare due to budget cuts. Real-terms spending on public health decreased by approximately 5\% between 2013/2014 and 2017/2018 and a recent review of the impact of financial pressures on healthcare services in the England highlighted that GUM services in particular are under

\section{Key messages}

- Symptoms of at least moderate depression and/or anxiety were reported frequently by women and heterosexual men attending genitourinary medicine (GUM) clinics in England.

- In women, depression symptoms and anxiety symptoms were associated with sexual risk behaviours including recent condomless sex with a non-regular partner and reporting multiple recent condomless sex partners.

- Psychological symptoms were associated with low selfefficacy for condom use among women and heterosexual men.

- The association between sexual and mental health should be considered both by general and GUM clinicians, and by commissioners of these services to ensure appropriate pathways are in place to support holistic care. considerable pressure as a result of budget reductions and increasing demand. ${ }^{27}$ Therefore, financial pressures pose a challenge to developing holistic pathways of care.

\section{CONCLUSION}

Our study demonstrates an association between depression symptoms and some measures of condomless sex and sexual risk among women and between anxiety symptoms and low self-efficacy for condom use among women and heterosexual men. Providers and commissioners of mental health and sexual health services should support the development of clinical care pathways which allow seamless referral between these services.

\section{Handling editor Richard Oliver de Visser}

Acknowledgements We thank all study participants for their time and effort. We gratefully acknowledge the contributions of all the AURAH clinic teams who helped with data collection, distribution of questionnaires and administrative tasks.

Collaborators The AURAH Study Group: Richard J Gilson, David Asboe, Nneka C Nwokolo, Amanda Clarke, Mark M Gompels, Sris Allan, Simon Collins, Fiona C Lampe, Christopher Scott, Sara Day, Martin Fisher, Jane Anderson, Rebecca O'Connell, Monica Lascar, Vanessa Apea, Paymaneh Farazmand, Susan Mann, Jyoti Dhar, Daniel R Ivens, Tariq Sadiq, Graham J Hart, Anne M Johnson, Alec Miners, Jonathan Elford, Nicky Perry and Michael Brady. AURAH Clinic Teams (in addition to study group members above): Barking: Rageshri Dhairyawan, Sharmin Obeyesekera; Barts: Vanessa Apea, John Saunders; Birmingham: Stephen Taylor, Gerry Gilleran, Cathy Stretton; Brighton: Martin Fisher, Amanda Clarke, Nicky Perry, Elaney Youssef, Celia Richardson, Louise Kerr, Mark Roche, David Stacey, Sarah Kirk; Bristol: Mark Gompels, Louise Jennings, Caroline Holder, Katie-Anne Baker; Calderdale \& Huddersfield: Maneh Farazmand, Matthew Robinson, Emma Street; Coventry: Sris Allan, Abayomi Shomoye; Dean Street: Nneka Nwokolo, Ali Ogilvy; Homerton: Jane Anderson, Sfiso Mguni, Rebecca Clark, Cynthia Sajani, Veronica Espa; John Hunter: David Asboe, Sara Day, Ali Ogilvy, Sarah Ladd; Kings: Susan Mann, Michael Brady, Jonathan Syred, Lisa Hamza, Lucy Campbell, Emily Wandolo, Janagan Alagarajah; Leicester: Jyoti Dhar, Linda Mashonganyika, Sally Batham; Mortimer Market: Richard Gilson, Rita Trombin, Ana Milinkovic, Clare Oakland; Newham: Rebecca O'Connell, Nyasha Makoka; Reading: Alan Tang, Ruth Wilson, Elizabeth Green, Sheila O'Connor, Sarah Kempster, Katie Keating-Fedders; Royal Free: Danie Ivens, Nicola Tyrrell, Jemima Rogers, Silvia Belmondo, Manjit Sohal; St George's: Tariq Sadiq, Wendy Majewska, Anne Patterson, Olanike Okolo, David Cox, Mariam Tarik, Charlotte Jackson, Jeanette Honigsbaum, Clare Boggon, Simone Ghosh, Bernard Kelly, Renee Aroney; The London: James Hand, Nyasha Makoka; West London Clinic for Sexual Health: Christopher Scott, Ali Ogilvy; Whipps Cross: Monica Lascar, Nyasha Makoka, Elias Phiri, Zandile Maseko. AURAH (Core) Study Group: Alison Rodger, Fiona Lampe, Andrew Phillips and Andrew Speakman. AURAH Data Managers: Andrew Speakman and Ada Miltz. AURAH Study Nurse Coordinator: Janey Sewell. CAPRA Advisory Board: Sir Nick Partridge, Kay Orton, Anthony Nardone, Ann Sullivan, Lorraine Sherr, Graham Hart, Simon Collins, Anne Johnson, Alec Miners and Jonathan Elford.

Contributors RMC, AR and FCL contributed to the study design, analysis and report writing. ARM and JS contributed to data handling, analysis and report writing. SM, JA, ROC, ANP, AS, AT, ML, RD, VA, AMJ, LS and SC contributed to the report writing.

Funding The AURAH study presents independent research funded by the National Institute for Health Research (NIHR) under its Programme Grants for Applied Research funding scheme (CAPRA: RP-PG-0608-10142). The views expressed in this publication are those of the authors and not necessarily those of the NHS, the NIHR or the Department of Health. The AURAH Study Group acknowledges the support of the NIHR through the Comprehensive Clinical Research Network.

\section{Competing interests None declared.}

\section{Patient consent for publication Not required.}

Ethics approval The research protocol and all versions of the study documents for the AURAH study were approved by the designated Research Ethics Committees (National Research Ethics Service Committee London-Hampstead, ref: 13/LO/0246). Based on these documents, the study received permission for clinical research at all participating National Health Service sites from local Research \& Development.

Provenance and peer review Not commissioned; externally peer reviewed.

Open access This is an open access article distributed in accordance with the Creative Commons Attribution Non Commercial (CC BY-NC 4.0) license, which permits others to distribute, remix, adapt, build upon this work non-commercially, and license their derivative works on different terms, provided the original work is 
properly cited, appropriate credit is given, any changes made indicated, and the use is non-commercial. See: http://creativecommons.org/licenses/by-nc/4.0/.

\section{REFERENCES}

1. National Collaborating Centre for Mental Health, 2010. Depression in adults with a chronic physical health problem. Available: www.bps.org.uk [Accessed 13 Sep 2018].

2. Katon WJ. Clinical and health services relationships between major depression, depressive symptoms, and general medical illness. Biol Psychiatry 2003;54:216-26.

3. Coulthard M, Farrell M, Singleton N, 2002. London: TSO report based on the analysis of the ONS survey of psychiatric morbidity among adults in Great Britain carried out in 2000 for the Department of health, the Scottish executive health department and the National assembly for Wales. Available: www.statistics.gov.uk

4. Moussavi S, Chatterii S, Verdes E, et al. Depression, chronic diseases, and decrements in health: results from the World health surveys. The Lancet 2007;370:851-8.

5. Field N, Prah P, Mercer CH, et al. Are depression and poor sexual health neglected comorbidities? Evidence from a population sample. BMJ Open 2016;6:e010521.

6. Osborn DPJ, King MB, Weir M. Psychiatric health in a sexually transmitted infections clinic: effect on reattendance. J Psychosom Res 2002;52:267-72.

7. Erbelding EJ, Hummel B, Hogan $T$, et al. High rates of depressive symptoms in STD clinic patients. Sex Transm Dis 2001;28:281-4.

8. Miltz AR, Rodger AJ, Sewell J, et al. Clinically significant depressive symptoms and sexual behaviour among men who have sex with men. BJPsych Open 2017;3:127-37.

9. Sewell J, Speakman A, Phillips AN, et al. A cross-sectional study on attitudes to and understanding of risk of acquisition of HIV: design, methods and participant characteristics. JMIR Res Protoc 2016;5:e58.

10. Kroenke K, Spitzer RL, Williams JB. The PHQ-9: validity of a brief depression severity measure. J Gen Intern Med 2001;16:606-13.

11. Spitzer RL, Kroenke K, Williams JB, et al. A brief measure for assessing generalized anxiety disorder: the GAD-7. Arch Intern Med 2006;166:1092.

12. Babor TF, Higgins-Biddle JC, Saunders JB. The alcohol use disorders identification test guidelines for use in primary care, 2001.

13. Zou G. A modified poisson regression approach to prospective studies with binary data. Am J Epidemiol 2004;159:702-6.

14. Stanfeld $S$, Clark C, Bebbington P. Chapter 2: Common mental health disorders. In: McManus S, Bebbington P, Jenkins R, eds. Mental health and wellbeing in England: Adult Psychiatric Morbidity Survey 2014. Leeds, 2016.
15. Bridges S. Mental health problems.. In: Mindell J, Craig R, eds. Health survey for England 2014. Leeds, 2015. http://healthsurvey.hscic.gov.uk/media/37739/HSE2014Ch2-Mental-health-problems.pdf.

16. Vasilenko SA, Kugler KC, Rice CE. Timing of first sexual intercourse and young adult health outcomes. J Adolesc Health 2016;59:291-7.

17. Pratt LA, Xu F, McQuillan GM, et al. The association of depression, risky sexual behaviours and herpes simplex virus type 2 in adults in NHANES, 2005-2008. Sex Transm Infect 2012;88:40-4.

18. Buffardi AL, Thomas KK, Holmes KK, et al. Moving upstream: ecosocial and psychosocial correlates of sexually transmitted infections among young adults in the United States. Am J Public Health 2008;98:1128-36.

19. Seth P, Patel SN, Sales JM, et al. The impact of depressive symptomatology on risky sexual behavior and sexual communication among African American female adolescents. Psychol Health Med 2011;16:346-56.

20. Zhan W, Shaboltas AV, Skochilov RV, et al. Depressive symptoms and unprotected sex in St. Petersburg, Russia. J Psychosom Res 2012;72:371-5.

21. Rubin AG, Gold MA, Primack BA. Associations between depressive symptoms and sexual risk behavior in a diverse sample of female adolescents. J Pediatr Adolesc Gynecol 2009;22:306-12.

22. Mazzaferro KE, Murray PJ, Ness RB, et al. Depression, stress, and social support as predictors of high-risk sexual behaviors and STIs in young women. J Adolesc Health 2006;39:601-3.

23. Chen Y, Wu J, Yi Q, et al. Depression associated with sexually transmitted infection in Canada. Sex Transm Infect 2008;84:535-40.

24. Jackson JM, Seth P, DiClemente RJ, et al. Association of depressive symptoms and substance use with risky sexual behavior and sexually transmitted infections among African American female adolescents seeking sexual health care. Am J Public Health 2015; 105:2137-42.

25. Khan MR, Kaufman JS, Pence BW, et al. Depression, sexually transmitted infection, and sexual risk behavior among young adults in the United States. Arch Pediatr Adolesc Med 2009;163:644.

26. Erez G, Pilver CE, Potenza MN. Gender-related differences in the associations between sexual impulsivity and psychiatric disorders. J Psychiatr Res 2014;55:117-25.

27. Robertson R, Wenzel L, Thompson J, et al, 2017. Understanding NHS financial pressures : how are they affecting patient care? Available: https://www.kingsfund.org uk/publications/understanding-nhs-financial-pressures [Accessed 13 Sep 2018]. 